\title{
INTERFACING MULTIPLEXED MICROFLUIDICS TO MALDI-MS BY HIGH-THROUGHPUT ROBOTIC SPOTTING

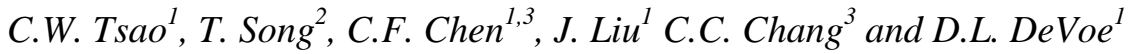 \\ ${ }^{1}$ Department of Mechanical Engineering, University of Maryland, College Park, MD, USA \\ ${ }^{2}$ Calibrant Biosystems Inc., Gaithersburg, MD, USA \\ ${ }^{3}$ Division of Mechanics, Research Center for Applied Science, Academia Sinica, Taipei, Taiwan
}

\begin{abstract}
Direct and automated spotting of analyte from multichannel microfluidic chips is demonstrated as a simple, robust, and highthroughput method for interfacing arrays of parallel microchannels with matrix-assisted laser desorption/ionization mass spectrometry (MALDI-MS). Using cyclic olefin copolymer (COC) chips containing 8 parallel $100 \mu \mathrm{m}$ x $46 \mu \mathrm{m}$ channels connected to a single input port, excellent spotting volume repeatability and MALDI-MS signal uniformity are achieved for a panel of sample peptides. Using the robotic spotting approach, chip-based reversedphase liquid chromatography (RPLC) separations are interfaced with nanofilament silicon LDI-MS, demonstrating the potential for this approach towards high-throughput on-chip bioanalysis coupled seamlessly with back-end mass spectrometry.
\end{abstract}

\section{INTRODUCTION}

The ability to perform high-throughput bioanalysis in a multiplexed platform is a substantial benefit promised by microfluidics technology. For applications which require analyte detection with high mass accuracy, interfacing microfluidics with mass spectrometry is a necessity. There are several examples of microfluidic systems containing multiplexed microchannels interfaced to electrospray ionization (ESI) tip arrays for ESI-MS analysis.[1-3] In practice, such systems are not well suited to parallel analysis since ESI-MS is an on-line method, with data acquisition performed in real-time. Coupling multiple electrospray tips with parallel MS analysis would thus require multiple MS tools, substantially limiting the practicality of this approach. A more compatible solution is to couple on-chip analyses with laser desorption/ionization methods such as MALDI-MS. In contrast to ESI-MS, MALDI-MS is an off-line soft ionization method in which sample is deposited onto a target plate and co-crystallized with a UV-absorbing organic matrix which aids in the ionization process during UV laser irradiation.[4] The off-line nature of MALDI-MS allows parallel deposition of analyte from multiplexed microchannels onto the MALDI target plate, followed by serial analysis of the deposited analyte spots. Because the laser spot size is typically on the order of $200 \mu \mathrm{m}$, closely spaced sample spots may be analyzed without concern for crosstalk, allowing dense microchannel arrays to be effectively interfaced to off-line MS.

A variety of approaches for interfacing microfluidic systems to MALDI-MS have been demonstrated.[5, 6] In a particular approach explored by Knapp et al., a multichannel cyclic olefin copolymer (COC) chip developed for parallel RPLC[7] was interfaced with a MALDI target through an electrically-mediated deposition technique[8] which was shown to be robust for the case of a 2-channel chromatography chip. Here we describe a simpler approach based on direct hydrodynamic contact spotting from multichannel microfluidic chips. Contact spotting provides a generic approach to LDI-MS interfacing which is straightforward to implement and integrate, robust even for large numbers of multiplexed microchannels, and compatible with a wide range of flow rates.

\section{HYRODYNAMIC CONTACT DEPOSITION}

The 8-channel chip design used in this work, shown in Figure 1(a), consisted of a symmetric 3-stage splitter connecting a single input port to an array of 8 parallel microchannels with $2 \mathrm{~mm}$ spacing. Each channel was nominally $100 \mu \mathrm{m}$ wide and $46 \mu \mathrm{m}$ deep, with geometric variances of less than $1.5 \%$ measured across all channels prior to chip bonding. The overall microchannel network was $50 \mathrm{~mm}$ long from input port to spotting tips.

Chip fabrication employed thermal embossing from a silicon master template and solvent bonding. After solvent bonding, the chip was shaped using a high precision CNC milling machine to produce regions with cross-sections $1 \mathrm{~mm}$ wide and $500 \mu \mathrm{m}$ thick surrounding each of the spotting tips, followed by a final cut to expose the fluid exit points using a semiconductor wafer dicing saw. Finally, a film of Teflon AF was deposited on the tip exit, thereby forming a hydrophobic surface to prevent fluid from wicking along the chip edge.

Two versions of the chip design were fabricated, one containing a methacrylate monolith within each spotting channel, and one without the monolith. Monoliths are highly porous inorganic or organic materials originally developed for conventional capillary HPLC separations. In comparison to packed columns, monoliths can offer fast and efficient separations using relatively low pressures, and by tuning the prepolymer composition and fabrication process, monoliths can be readily adapted for different separation mechanisms without further treatments. One of the most attractive characteristics of monolithic materials, especially the polymethacrylate/acrylate types, is that their photopolymerization-based synthesis enables the preparation of well-defined monoliths within specific regions of microchannel networks. The monolith was fabricated by first modifying the COC microchannel surface use a photografting method to enhance monolith/wall adhesion. The reversed-phase monolith was then fabricated in situ using a UV photolithography process first described by Frechet et al [9], resulting in a monolith with a morphology well suited for application to liquid chromatography applications.

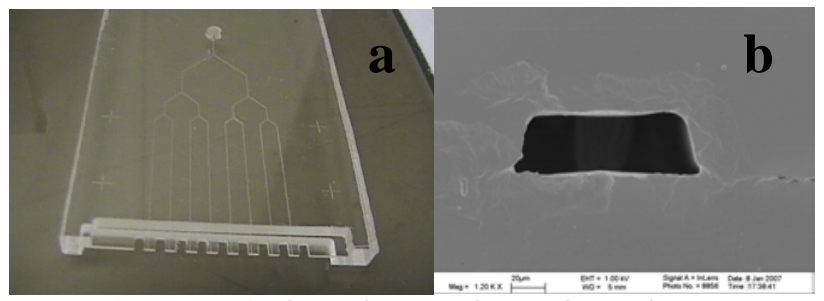

Figure 1: (a) An 8 channel COC chip with on-chip spotting tips connected to a single flow source, and (b) electron micrograph showing a typical spotting tip exit surface following chip dicing.

Custom microstructured MALDI target plates were fabricated by first spin-coating a thin Teflon AF layer onto an optically- 
smooth stainless steel plate. The Teflon AF film, approximately 1 $\mu \mathrm{m}$ thick, was baked at $250{ }^{\circ} \mathrm{C}$ for $2 \mathrm{hr}$ to produce a dense hydrophobic layer over the entire stainless steel surface. A $300 \mathrm{~nm}$ thick gold film was then deposited by e-beam evaporation using a shadow mask to pattern four $8 \times 8$ arrays of $250 \mu \mathrm{m}$ diameter gold pads on top of the Teflon AF film.

The experimental system used for spotting tests is shown in Figure 2. The chip inlet was interfaced to a syringe pump through a $100 \mu \mathrm{m}$ i.d. capillary using an Upchurch Nanoport connector. The chip was mounted to a single-axis positioning stage to control the displacement of the chip relative to the target plate, which was mounted to a seperate two-axis stage. The on-chip deposition tips were aligned to a first row of gold anchor pads and positioned 2 $\mathrm{mm}$ from the target surface. Following initial positioning, all control over the deposition process was automated using custom LabView software which controlled positioning of the stages through micro-stepper motors with a resolution of $5 \mu \mathrm{m}$.

Automatic spotting was performed by moving the chip 200 $\mu \mathrm{m}$ from the target surface to transfer droplets to the gold pads, then returning to the initial $2 \mathrm{~mm}$ gap and indexing the stage for the next row. Sequential images of the robotic spotting process are shown in Figure 3. Each of the 8 channels deposited droplets in parallel onto the MALDI target plate, with a total of 8 spotting events to fill the array. For each experiment, peptide sample premix with matrix solution was deposited onto 64 target spots in an $8 \times 8$ array.

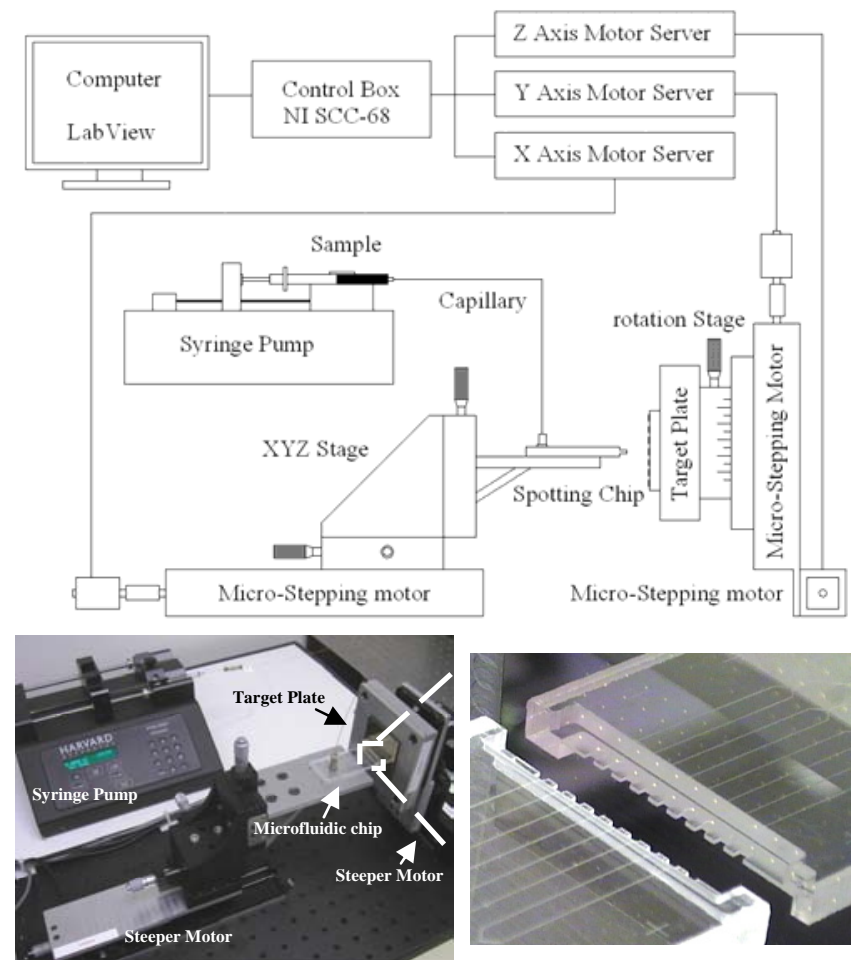

Figure 2: Experiment set of the robotic spotting system, with the multiplexed microfluidic chip and MALDI target fixed and aligned on their respective robotic stages.

The average droplet volume and coefficient of variation (CV) transferred from the COC chip to the MALDI target substrate is shown in Figure 4. The chip containing monoliths for liquid chromatography applications showed a CV of $12.9 \%$ for multiple droplets from a single channel, and up to $7.0 \%$ for single spotting events across all 8 channels. This compares favorably with values of $34.7 \%$ and $25.2 \%$ from a single channel and across all channels, respectively, without monolith inside the multiplexed channel array. [10]

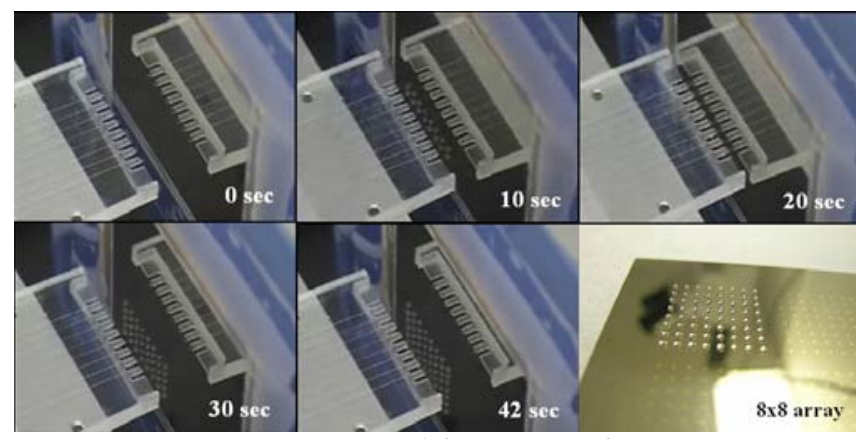

Figure 3: Consecutive images of the automated contact spotting process using a $12 \mu \mathrm{L} / \mathrm{min}$ total flow rate and 6 sec interval between spotting events, resulting in an $8 \times 8$ array of $150 \mathrm{~nL}$ droplets deposited on the microsctructured MALDI target surface.

Following spotting, the array of droplets was air dried, leaving co-crystallized sample and matrix on the gold anchors. The hydrophilic anchor pads were found to be effective in encouraging the efficient transfer of droplets from the hydrophobic chip spotting tips. The anchor pads also served to maintain the positions of the deposited droplets once transferred to the MALDI target. Figure 5 shows sequential images of the solvent evaporation and sample/matrix co-crystallization process on a single gold anchor pad.

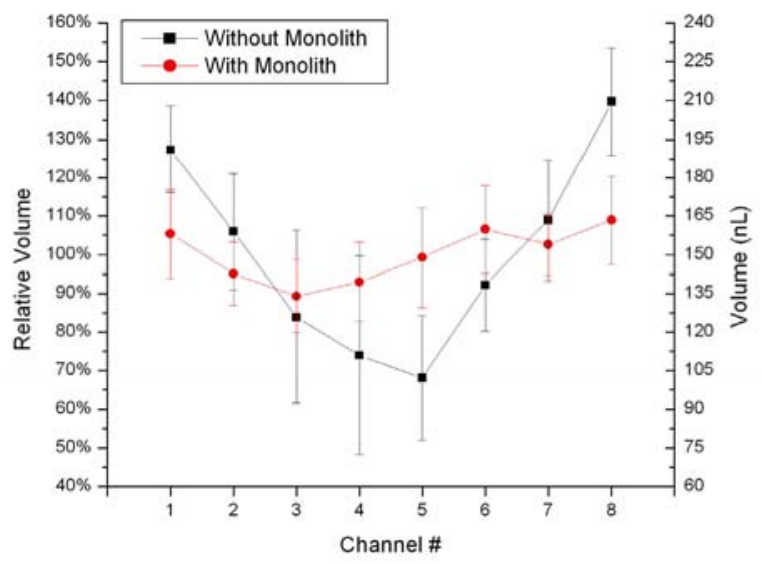

Figure 4: Average droplet volume transfer based on 8 consecutive spot runs comparing channels with and without monoliths.



Figure 5: Sequential images showing the process of solvent evaporation and sample/matrix co-crystallization on a gold target anchor.

\section{MULTIPLEXED MALDI-MS MEASUREMENTS}

Uniformity of MALDI-MS measurements using the spotting system was characterized with a panel of model peptides consisting of RASG-1 (MW 1000.5), angiotensin fragment 1 (MW 898.7), angiotensin II (MW 1045.5), bradykinin (MW 1059.6), 
angiotensin I (MW 1295.7), renin substrate (MW 1758.0), enolase T35 (MW 1872.0), enolase T37 (MW 2827.3), and melittin (MW 2845.7). Sample was mixed with a MALDI matrix solution consisting of $10 \mu \mathrm{g} / \mu \mathrm{L} \alpha$-cyano-4-hydroxycinnamic acid (CHCA) mixed into acetonitrile, DI water, and acetic acid 5:4:1 v/v/v. The matrix solution was mixed with the peptide sample $1: 1 \mathrm{v} / \mathrm{v}$, for final peptide concentrations of $0.5 \mathrm{pmol} / \mu \mathrm{L}$.



Figure 6: MALDI-MS spectra for sample deposited from all 8 channels during a single spotting event, revealing excellent repeatability
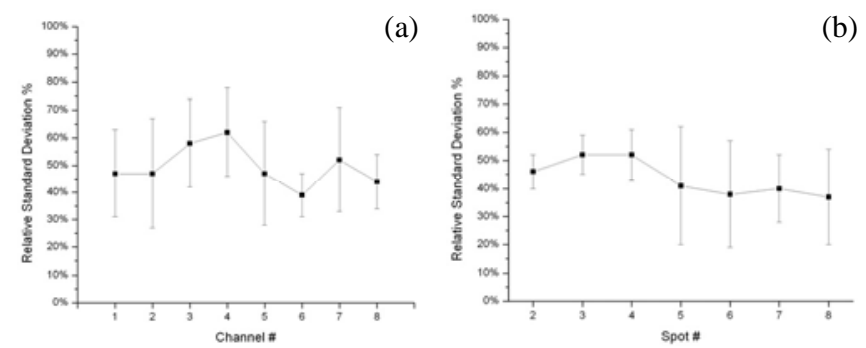

Figure 7: Uniformity of MALDI-MS signal (a) between different spotting events averaged over all channels, and (b) between different channels averaged over all spotting events.

Except for the $\mathrm{m} / \mathrm{z}$ value at 1000.5 corresponding to RASG-1, which presents a relatively low signal intensity with a signal-tonoise ratio $(\mathrm{S} / \mathrm{N})$ less than 3, MALDI-MS spectra acquired from the 64 sample spots provided repeatable identification for 8 of the 9 model peptides for all spotting events. Figure 6 shows the mass spectra from all 8 channels during a single spotting event. The MALDI-MS measurements were performed on a Kratos Amixa time-of-flight (TOF) mass spectrometer. All LDI-MS spectra were acquired in linear, positive ion mode using between 25 and 50 laser pulses from a $337 \mathrm{~nm}$ nitrogen laser with a 3 ns pulse width. Laser energy was optimized based on signal-to-noise ratio using a test sample spot, and held constant during all experiments. The resulting uniformity of MALDI-MS signal intensities is displayed in Figure 7. Figure 7(a) shows the deviation of intensity values between different spotting events averaged over all channels, while Figure 7(b) reveals deviations between each channel averaged over all spotting events. Compared with traditional spotting methods, which can often result in $>100 \%$ variations in signal intensity, the measured RSD values are encouraging.

\section{INTERFACING CHIP-RPLC WITH LDI-MS}

The key to achieving highly repeatable droplet generation from the 8-channel spotting chip was to integrated monoliths into the spotting channels, thereby reducing the effects of capillary forces on the relative flow rates among the channels within the array. This fact makes the spotting approach attractive for multiplexed RPLC separations, since monoliths can provide excellent stationary phases for a range of chromatographic separations, including RPLC.

The RPLC chip design used here is shown in Figure 8. The chip consists of a sample injection channel, an LC pump connection, and a single serpentine RPLC separation channel containing a $15 \mathrm{~cm}$ long monolith. Each channel was nominally $100 \mu \mathrm{m}$ wide and $46 \mu \mathrm{m}$ deep, with geometric variances of less than $1.5 \%$ measured across all channels prior to chip bonding. Chip fabrication employed thermal embossing from a silicon master template and solvent bonding using procedures identical to previously-reported process. After solvent bonding, the chip was cut to expose the fluid exit points using a semiconductor wafer dicing saw. Because high pressures are used during RPLC separation, surgical stainless steel needles were used as high pressure and low dead-volume interconnections between off-chip components and the on-chip sample injector and separation channels.

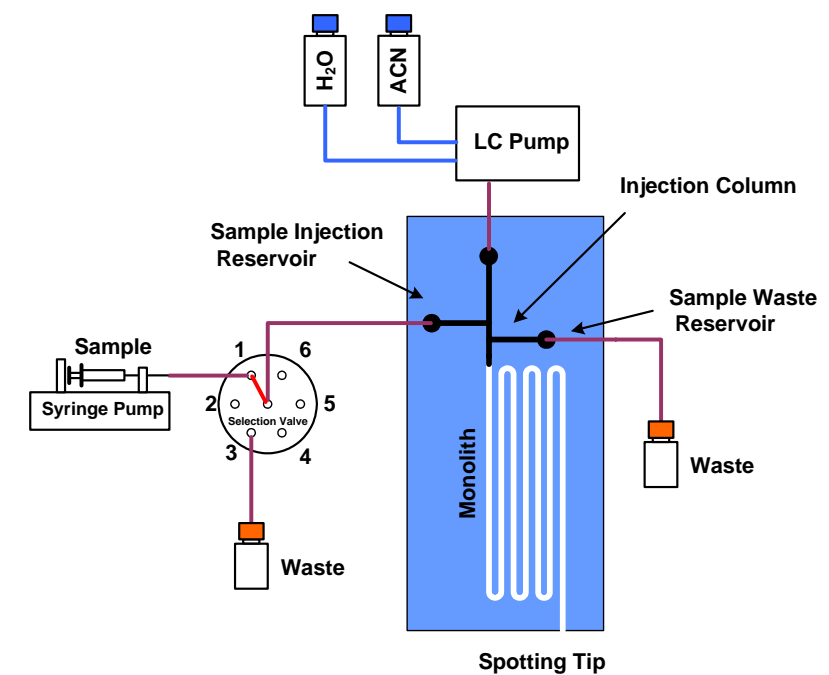

Figure 8: LC/MS chip design and experiment setup

Unlike the previous MALDI-MS characterization results (Figs. 6-7), which were generated using a traditional stainless steel MALDI target during deposition and analysis (Figs. 6-7), the RPLC separations were interfaced with a nanofilament silicon (nSi) target which eliminates the need for organic matrix to be added to the sample [11]. The nSi substrates were fabricated by a metal-assisted oxidative silicon etching process.[12] A $3 \mathrm{~nm}$ thick gold layer was deposited onto silicon wafer by e-beam evaporation followed by immersion into a solution of $\mathrm{HF}, \mathrm{H}_{2} \mathrm{O}_{2}$, and $\mathrm{EtOH}$ $(1: 1: 1 \mathrm{v} / \mathrm{v} / \mathrm{v})$ in a teflon beaker for $32 \mathrm{~s}$. The etched wafer was oxidized by a $30 \mathrm{~min}$ UV/ozone treatment (PSD-UV, Novascan Technologies, Ames, IA) and the oxidized surface was then fluorinated by (pentafluorophenyl)propyldimethylchlorosilane. 
To evaluate the system, model peptides consisting of [leu5]enkephalin (MW 555.62), angiotensin II (MW 1046.18), bradykinin (MW 1060.61) and neurotensin (MW 1672.92) were first dissolved in $10 \mathrm{mM}$ sodium carbonate/bicarbonate buffer $(\mathrm{pH}$ 9.0 ) to a concentration of $2 \mathrm{mM}$. Equal volumes of each peptide solution were mixed and then diluted 5-fold with HPLC grade water before being injected into the microfluidic chip. Separation of peptides on the $15 \mathrm{~cm}$ long monolith was achieved using a piecewise linear acetonitrile (ACN) solvent gradient, with an initial gradient of $5 \%$ to $20 \%$ ACN during the first $15 \mathrm{~min}$, followed by $20 \%$ to $50 \%$ ACN over the next 5 min, and a constant $50 \%$ ACN concentration for the remainder of the separation. The total flow rate was set at $1.5 \mathrm{~mL} / \mathrm{min}$ using the LC pump, with the on-chip splitter delivering a flow rate of $0.2 \sim 0.5 \mu \mathrm{L} / \mathrm{min}$ through the monolith-filled separation channel.

Throughout the separation, automatic spotting was performed by moving the chip $200 \mu \mathrm{m}$ from the target surface to transfer droplets to the nanofilament substrate, then returning to the initial $2 \mathrm{~mm}$ gap and indexing the stage for the next spot. A total of 20 droplets were eluted onto the nSi substrate, with 2 min between each spotting event over the full $40 \mathrm{~min}$ separation time. The resulting mass spectra measured from each of the 20 deposited spots is shown Figure 9. Each of the sample peptides was observed in specific fractions deposited on the nSi surface, with [leu5]enkephalin identified from spots \#2 and \#3, angiotensin II from spot \#5 and bradykinin and neurotensin together from spot \#10. The co-elution of [leu5]-enkephalin across two adjacent fractions indicates a relatively large bandwidth for this peptide, while the simultaneous elution of bradykinin and neurotensin within a single fraction suggests that more fractions should be sampled to fully resolve these components with similar hydrophobicity.

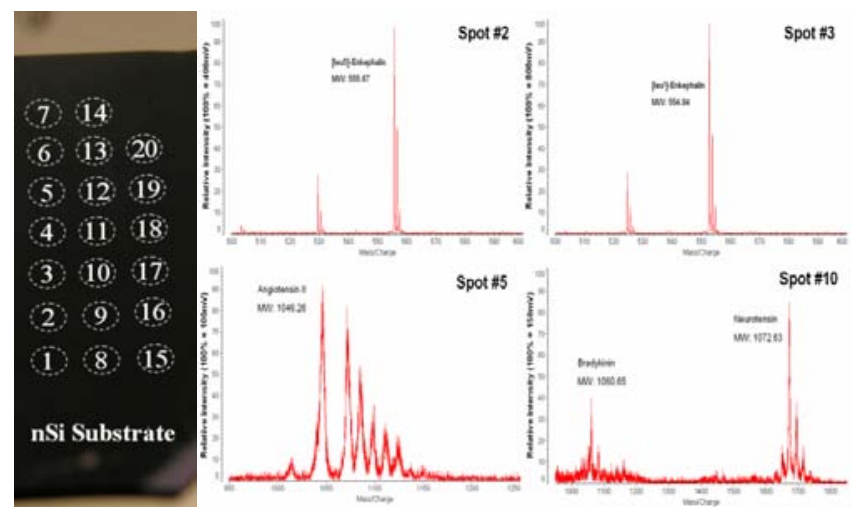

Figure 9: Nanofilament LDI-MS spectra from four nSi target spots containing peptides separated by the microfluidic chip-RPLC system.

\section{CONCLUSION}

Automated contact spotting is an exceptionally simple and robust approach for coupling microfluidic separations to off-line LDI-MS mass spectrometry. Spotting from multiplexed arrays of parallel microchannels revealed good uniformity in both droplet volume and MALDI-MS signal intensity across all channels and multiple spotting events, while spotting from a single separation channel onto an $\mathrm{nSi}$ LDI-MS target demonstrated that hydrodynamic contact spotting may be effectively coupled with microfluidic RPLC separations. Further work to demonstrate parallel RPLC interfaced to nSi LDI-MS as an approach to highthroughput bioanalysis leveraging the unique benefits of microfluidic technology is ongoing.

\section{REFERENCES}

[1] Q. F. Xue, F. Foret, Y. M. Dunayevskiy, P. M. Zavracky, N. E. McGruer, and B. L. Karger, "Multichannel microchip electrospray mass spectrometry," Analytical Chemistry, vol. 69, pp. 426-430, 1997.

[2] K. Q. Tang, Y. H. Lin, D. W. Matson, T. Kim, and R. D. Smith, "Generation of multiple electrosprays using microfabricated emitter arrays for improved mass spectrometric sensitivity," Analytical Chemistry, vol. 73, pp. 1658-1663, 2001.

[3] S. Le Gac, S. Arscott, C. Cren-Olive, and C. Rolando, "Twodimensional microfabricated sources for nanoelectrospray," Journal of Mass Spectrometry, vol. 38, pp. 1259-1264, 2003.

[4] M. Karas and F. Hillenkamp, "Laser Desorption Ionization of Proteins with Molecular Masses Exceeding 10000 Daltons," Analytical Chemistry, vol. 60, pp. 2299-2301, 1988.

[5] F. Foret and J. Preisler, "Liquid phase interfacing and miniaturization in matrix-assisted laser desorption/ionization mass spectrometry," Proteomics, vol. 2, pp. 360-372, 2002.

[6] D. L. DeVoe and C. S. Lee, "Microfluidic technologies for MALDI-MS in proteomics," Electrophoresis, vol. 27, pp. 3559-3568, 2006.

[7] K. W. Ro, J. Liu, and D. R. Knapp, "Plastic microchip liquid chromatography-matrix-assisted laser desorption/ionization mass spectrometry using monolithic columns," $J$. Chromatography A, vol. 1111, pp. 40-47, 2006.

[8] C. Ericson, Q. T. Phung, D. M. Horn, E. C. Peters, J. R. Fitchett, S. B. Ficarro, A. R. Salomon, L. M. Brill, and A. Brock, "An automated noncontact deposition interface for liquid chromatography matrix-assisted laser desorption/ionization mass spectrometry," Analytical Chemistry, vol. 75, pp. 2309-2315, 2003.

[9] T. B. Stachowiak, T. Rohr, E. F. Hilder, D. S. Peterson, M. Q. Yi, F. Svec, and J. M. J. Frechet, "Fabrication of porous polymer monoliths covalently attached to the walls of channels in plastic microdevices," Electrophoresis, vol. 24, pp. 3689-3693, 2003.

[10] C. W. Tsao, J. Liu, and D. L. DeVoe, "Droplet formation from hydrodynamically coupled capillaries for parallel microfluidic contact spotting," Journal of Micromechanics and Microengineering, vol. 18, pp. 025013, 2008.

[11] C. W. Tsao, P. Kumar, J. Liu, and D. L. DeVoe, "Dynamic electrowetting on nanofilament silicon for matrix-free laser desorption/ionization mass spectrometry," Analytical Chemistry, 2008, In press.

[12] X. Li and P. W. Bohn, "Metal-assisted chemical etching in HF/H2O2 produces porous silicon," Applied Physics Letters, vol. 77, pp. 2572-2574, 2000. 